\title{
COVID-19 enabled co-authoring networks: a country-case analysis
}

\author{
E. Sachini ${ }^{1} \cdot$ K. Sioumalas-Christodoulou ${ }^{1}$ (D) . C. Chrysomallidis ${ }^{1} \cdot$ G. Siganos ${ }^{1}$. \\ N. Bouras ${ }^{1} \cdot$ N. Karampekios ${ }^{1}$
}

Received: 2 November 2020 / Accepted: 11 March 2021 / Published online: 26 March 2021

(c) Akadémiai Kiadó, Budapest, Hungary 2021

\begin{abstract}
In this paper we seek to examine the co-authoring pattern of a select group of researchers that are affiliated with a specific country. By way of making use of standard bibliometric analysis, we explore the publication evolution of all COVID-19-related peer reviewed papers that have been (co)-authored by researchers that are affiliated with Greek institutions. The aim is to identify its advancement over time, the institutions involved and the countries with which the co-authors are affiliated with. The timeframe of the study spans from the moment that WHO Director-General declared the novel coronavirus outbreak a public health emergency of international concern (WHO, 2020. Archived: WHO timelinecovid-19. Retrieved from Archived: Who Timeline-COVID-19. https:/www.who.int/news/ item/27-04-2020-who-timeline---covid-19. Accessed on 10 May 2020., Archived: WHO timeline-covid-19), January 2020, to October 2020. Findings indicate that there is a steady increase in the number of publications as well as the number of scientific collaborations over time. At a cross-country level, results suggest that the affiliated institutional sectors such as the Higher Education Sector (HES) and the Government Sector (GOV) contributed the most in terms of scientific output. On an international scale, the evolution of the scientific collaboration is imprinted and distributed as a chain of affiliations that linked nations together. Such chains are represented as clusters of countries, in which the scientific connections between different countries can be visualised. It can be reasoned that a significant amount of publications (20\%) is affiliated with countries having "traditionally" major scientific impact on the field of Medicine.
\end{abstract}

Keywords COVID-19 · Co-authorship · Scientific collaboration · Bibliometrics $\cdot$ Greece

\section{Introduction}

Responding to the COVID-19 pandemic requires a fully-blown science-based approach. This has been the case so far. Policy makers, researchers and funders alike have mobilized to come up with a solution that puts a stop to the devastating effects of this coronavirus.

K. Sioumalas-Christodoulou ksioumalas@ekt.gr

1 National Documentation Centre, Athens, Greece 
This global-scale, war-like mobilization is a corresponding measure to the detrimental consequences of the pandemic, foremost in the form of human casualties, the significant and long-term health problems for those recovering and the important social and economic side-effects from lockdowns and social distancing rules, such as income and job loss, in turn, directly affecting well-being (Bonaccorsi et al., 2020).

Scientists corresponding to the Medical and Health Sciences (Frascati manual) have been at the forefront of attempting to come up with a solution against COVID-19. Yet, it would do injustice not to mention that this pivot towards addressing the results of this coronavirus (and the attempts to mitigate its downsides) has not been the case for all other research communities. To name just a very few: social and behavioural scientists have attempted to examine the impact on productivity (Dingel \& Neiman, 2020), employment (Fana et al., 2020), fiscal policies, inequality (Blundell et al., 2020), while engineers have been quick to address the challenges posed by the pandemic. In the latter case, the field has reoriented itself towards addressing the pandemic. To name just a very few cases, see, for example, in the case of electrical engineering (Paaso et al., 2020) as well as mechanical (Botenga, 2020). Similarly, reporting on how mega-technological scientific fields (e.g. artificial intelligence (OECD, 2020a), digital education (OECD, 2020b) can contribute into achieving this objective/goal have been rising steadily.

It should be noted, however, that this cumulative effect is not without its downsides. Indeed, this has already been identified in the case of patent and litigation filings where individuals and/or organisations scramble to take positions in the vaccine race in the postCOVID-19 world (Fraizer \& Auvil, 2020) even when policy makers (Botenga, 2020) are calling for vaccines to be considered as global public goods with intellectual property on coronavirus vaccines be shared freely (Nature, 2020). A related concern points to the direction of COVID-19 research dwarfing every other field of research. While this, obviously, should be attributed to the need to come up with a solution against the raging pandemic in a timely fashion, this, potentially, can lead to long term imbalances among scientific fields (Pai, 2020; Prudêncio \& Costa, 2020).

Concerning research as a distinct policy field, a number of COVID-19-induced modifications can be identified from the usual conduct. For one, huge, urgent and with less strings attached to them sums of funds are being expediently provided by the public sector (EU Commission, 2020). In most cases, these are coupled by private funding sources of the same magnitude (ScienceBusiness, 2020a, b). Similarly, central public innovation-related initiatives (e.g. public sector innovation) are being modified to fit the need to address the pandemic (OPSI, 2020). A second one refers to the time from submission to acceptance and subsequent publication of peer reviewed articles. As the only measure to boost the research validity of any potential breakthrough against the pandemic, editorial practises have been significantly diminished in terms of the necessary time to proceed to the review process (Horbach, 2020). One could only hope that potential mishaps (Collins et al., 2020) due to the cutting down of methodological corners is just a rare phenomenon.

Responding against COVID-19 has been enabled by existing trends in the conduct of science policy. First and foremost has been global research collaboration. With one out of five research articles having authors from more than one country (NSB, 2019), science outputs, such as publications, have become a global undertaking involving scholars from different countries and environments (Wagner \& Graber, 2018). Indeed, this pays out since internationally-authored publications attract a greater number of citations as opposed to publications that have been authored by exclusively domestically-based researchers (NSB, 2019). COVID-19 only increased this trend (Zhang et al., 2020; Fry et al., 2020; Homolak et al., 2020). While the response is actually in the making and thus the monitoring of the 
global partnerships that have been initiated as a result of the pandemic is a current phenomenon not yet settled, past pandemics can provide lessons (Lee \& Haupt, 2020). This is closely associated with another existing science-related trend-open science. Free distribution of research in the interest of strong science and the public good has only been made an even higher objective during the pandemic in the attempt to remove obstacles to the free flow of research data and ideas, and thus accelerate the pace of research critical to combating the disease (OECD, 2020c).

Indeed, this emphasis by scientists on all things COVID-19-related has been observed in a number of secondary studies that examine the rate of science outputs. Bibliometrics and the overall study of examining the evolution of science is important in that it helps understand the rate, priorities of science and helps document this systemic approach to help curb COVID-19 (Benjamens et al., 2020). These analyses bring testament to this scientific pivot. For example, bibliometric studies (Palayew et al., 2020; Hossain, 2020; Kambhampati et al., 2020) document this steep increase in terms of science outputs during and as a direct result of this pandemic. It is on this strand of the existing bibliography that this paper stands upon. Specifically, by way of making use of bibliometric approaches, we seek to explore the bibliographic performance relating to COVID-19 of a select group of researchers that are located (affiliated) within a specific country. In terms of the existing bibliometric bibliography, rather than seeking to identify the trends in a global scale or the growth patterns of closed versus open publications, we zoom in on a country case. Rather than a rupture with existing bibliography, this would shed light on the performance capacity of a national research system against a ranging pandemic, all the more so since reacting to the pandemic has been, first and foremost, a public policy task at the national level.

\section{Objective and Scope}

The objective of this paper is to identify and explore the COVID-19 related scientific publications (co)authored by researchers that are affiliated with tertiary education and/or research centers located in Greece (hereafter abbreviated as "COVID-19 related Greek publications"). By the term 'identify and explore', it should be taken to mean to inquire and provide a range of relevant metrics that would help understand the productivity of this group of researchers over time as well as its collaborative pattern. The idea behind the provision of such metrics is to enable the authors to determine the response of the scientific community throughout the pandemic - as measured through the production of scientific publications, its number, etc. as well the researcher's network.

The scope of this paper is built around the field of bibliometrics and its endeavour to explore the metadata of the COVID-19 related Greek publications. Focusing on the scientific publications produced by researchers (herein, the concept is considered identical to scholars, scientists, authors), we aim to describe the level of academic activity that the need to understand and come up with an appropriate answer against this pandemic has been initiated. Focusing on a country-case, the paper seeks to examine the number and rate of such academic output (i.e. publications) over time. Moreover, this paper seeks to identify the collaborative networks in which these authors have been participating in to produce this scientific output. Importantly, the identification of these networks takes into consideration the parameter of time and, thus, identify the evolution of the network from the beginnings of the pandemic to October 2020. 


\section{Structure}

The structure of the paper is as follows.

The next section ("Methodology") outlines the methodology followed and the target population. It, also, describes the process of collection, retrieval and validation of the data and variables upon which the analysis is based. Section "Analysis" unfolds the conducted bibliometric analysis' approaches. It is followed by the "Findings" section where descriptive statistics on the COVID-19 related Greek scientific publications and affiliations, as well as graphs concerning the depiction of international scientific collaboration between the affiliated countries are laid out. Additionally, insight into the main outcomes of the object of study, including the national scientific collaboration level and the international collaboration among the affiliated countries over time, is provided. This is succeeded by the discussion where the findings are contextualised and set within specific considerations. Last is the formulation of points for further research.

\section{Methodology}

In this section, the steps towards identifying the population are described, the bibliometric data collection process is laid out and the variables' selection process is presented.

\section{Target population}

This paper has a strong national focus - that is, it explores the research performance of a specific population group of researchers located (affiliated) within Greek institutions. There are a number of reasons for focusing on a country-level analysis. First of all, this is a valid bibliometric research strategy, inasmuch bibliometrics analyze the scienceoutput performance of, among others, national innovation systems. Secondly, Greek science stands as a competitive research ecosystem. This is attested, for example, a systemic over-performance in competitive European collaborative projects (Horizon, 2020) and an increasingly good bibliometric performance (Sachini et al., 2020b). In addition, Greek highly educated individuals (Sachini et al., 2020a) occupy a disproportionately high number of academic and research positions in US' IVY league universities (Yuret, 2017). Indeed, its human capital to be this mobile, it stands to reason for this paper to examine the collaborative pattern that has been initiated by Greek researchers during and as a direct result to the COVID-19 pandemic.

There are a number of wider reasons supporting the national case. One is that Greece has fared really well in mitigating the effects of the pandemic (Mariolis et al., 2020). This is mostly attributed to policy makers that gave the front seat to the medical experts that had had a significant leverage into the everyday policy directions. This was translated into a list of proactive, lockdown measures, that were, also, taken in view of the decade-long economic crisis taking its toll into the public health system. As such, one would be willing to examine how the science system of Greece responded to the COVID-19 pandemic in terms of the coronavirus-related science output. 
Table 1 COVID-19 related queries for a range of bibliometric sources

\begin{tabular}{|c|c|}
\hline Scopus & $\begin{array}{l}\text { TITLE-ABS-KEY }(* \text { covid-19) OR TITLE-ABS-KEY }(* \text { COVID19) OR TITLE-ABS- } \\
\text { KEY }(* \text { COVID2019) OR TITLE-ABS-KEY (*SARS-CoV-2) OR TITLE-ABS-KEY } \\
(* 2019-n C o V) \text { OR TITLE-ABS-KEY }(* 2019 \text { coronavirus) OR TITLE-ABS-KEY } \\
(* \text { coronavirus disease } 2019) \text { AND AFFILCOUNTRY ( Greece) }\end{array}$ \\
\hline WoS & $\begin{array}{l}(\mathrm{TS}=\text { covid }-19 \text { OR } \mathrm{TS}=\mathrm{COVID} 19 \mathrm{OR} \mathrm{TS}=\mathrm{COVID} 2019 \text { OR } \mathrm{TS}=\mathrm{SARS}-\mathrm{CoV}-2 \mathrm{OR} \\
\mathrm{TS}=2019-\mathrm{nCoV} \text { OR TS }=2019 \text { coronavirus OR TS }=\text { coronavirus disease } 2019) \\
\text { AND CU }=\text { Greece }\end{array}$ \\
\hline Pubmed & $\begin{array}{l}\text { (covid-19 OR COVID19 OR COVID2019 OR SARS-CoV-2 OR 2019-nCoV OR } 2019 \\
\text { coronavirus OR coronavirus disease 2019) AND (Greece[Affiliation]) }\end{array}$ \\
\hline Pubmed Central & $\begin{array}{l}\text { (covid-19 OR COVID19 OR COVID2019 OR SARS-CoV-2 OR 2019-nCoV OR } 2019 \\
\text { coronavirus OR coronavirus disease 2019) AND (Greece[Affiliation]) }\end{array}$ \\
\hline
\end{tabular}

Table 2 Identified COVID-19 related Greek publications in each bibliometric database

\begin{tabular}{lllll}
\hline Bibliometric database & Scopus & WoS & Pubmed & Pubmed Central \\
\hline Publications & 570 & 423 & 601 & 600 \\
Publications with DOI & 561 & 419 & 591 & 584 \\
$\begin{array}{c}\text { Publications with Pub- } \\
\text { medID/PMCID }\end{array}$ & 426 & 402 & 601 & 600 \\
\hline
\end{tabular}

For document matching, DOI and PubmedID/PMCID were complementary utilised

\section{Data collection}

A two-stage process was used to collect the bibliometric data of the COVID-19 related Greek publications. Regarding the first stage, a query process was performed in order to identify the target population. At a second stage, within the context of the data collection, certain variables relevant to the researcher profile were retrieved from the Pubmed database for analysis purposes.

Regarding the first stage, four bibliometric databases were examined. Scopus, Web of Science (WoS), Pubmed (PM) and Pubmed Central (PMC). With respect to Web of Science database the core collection indices SCIE, SSCI and AHCI were utilised. The aforementioned databases are widely considered to be the most comprehensive bibliometric datasets, worldwide (Zhu \& Liu, 2020). Aware of potential discrepancies between the databases (Kokol \& Vošner, 2018; Mercer University, 2020), this paper followed a multipronged approach. Queries relevant to the object of study were used as input in each bibliometric database. Following Kousha and Thelwall (2020), the queries were designed to be as inclusive as possible for the database in terms of document type and part of the document searched. Specifically, the queries included full text, when available, otherwise all metadata fields (e.g., title, abstract, keywords). Although the publication year is not predefined, given the obvious emphasis on COVID-19, the queries included the most recent research focusing on the issue, assuming that it includes the current official disease description (see Table 1).

The combined queries did function in all bibliometric databases. Table 2 shows the identified publications in each bibliometric database. As publications, the results included reviews, letters, articles, notes, editorials and conference papers. For each publication, the DOI, Pubmed ID, date of publication (where plausible), author names, author affiliation 
Table 3 Uniquely identified publications in terms of DOI and Pubmed ID

\begin{tabular}{lll}
\hline Variables & Removal of duplicate documents & $\begin{array}{l}\text { Unique } \\
\text { docu- } \\
\text { ments }\end{array}$ \\
\hline DOI & $\begin{array}{c}\text { Scopus } \cup \text { WoS } \\
\text { pus } \cap \text { WoS } \cap P M \cap P M C-(\text { Sco- }\end{array}$ & 647 \\
Pubmed ID & $\begin{array}{c}\text { Scopus } \cup \text { WoS } \cup P M \cup P M C-(S c o- \\
\text { pus } \cap \text { WoS } \cap P M \cap P M C)\end{array}$ & 9 \\
\hline
\end{tabular}

(where plausible) and document title were downloaded. Obviously, some of the identified publications did appear in more than one bibliometric database. To address that issue and thus obtain unique documents, the DOI and the Pubmed ID of each document were complementary utilised. Particularly, the DOI, as a unique identifier for each document was used for matching documents (Visser et al., 2020). Whenever a DOI was missing the Pubmed ID of each document was utilised in a similar manner. In total, 663 unique publications-in terms of DOI and Pubmed ID were identified (see Table 3). Scopus recorded 42 distinct documents, WoS 18 documents and PMC 1 document (see Eqs. 1-3). Pubmed had the best coverage indexing 602 publications. This can be potentially attributed to the fact that Pubmed indexes solely records of biomedical literature and relevant content on life sciences.

At this point, additional cross-checking of the results with the rest of the downloaded variables was performed. The document title, author names, author affiliation and date of publication were sequentially examined for duplicate values. In total, 7 documents were found having the same title and thus removed. ${ }^{1}$ As a result, the final dataset consisted of 656 uniquely identified publications (647 from DOI and 9 from Pubmed ID intersections, see Table 3).K

$$
\begin{aligned}
& (\mathrm{WoS} \cup \mathrm{PM} \cup \mathrm{PMC})^{c} \cap \mathrm{Scopus}=42 \\
& (\text { Scopus } \cup \mathrm{PM} \cup \mathrm{PMC})^{c} \cap \text { WoS }=18 \\
& (\text { Scopus } \cup \text { WoS } \cup \mathrm{PM})^{c} \cap \mathrm{PMC}=1
\end{aligned}
$$

\section{Variables}

As the second stage, the retrieval of the DOI's as well as Pubmed IDs enabled the research and allowed the locating and downloading of information relevant to researchers' bibliographic profile.

Bibliometric analysis can yield different types of information depending on the bibliometric indicators used. The main objective of the analysis is to identify and explore the GC related publications in terms of (a) determining the response of the scientific community throughout the pandemic (b) enhancing our understanding of the international scientific collaboration networks as well as (c) the relevant institution categories' contribution across

\footnotetext{
1 This occurred due to the fact that although Pubmed ID's have different values, they sometimes refer to the same document.
} 
different sectors (HES, GOV, BES, PNP). Accordingly, complementary to the documents' DOI and Pubmed ID, the following variables were selected to be retrieved: author names, date of document publication, author (co)affiliation.

As indicated in the previous subsection ("Target population"), not all bibliometric databases support the extraction of the"date of publication" as a feature variable of a document found therein. For example, Scopus does not offer this option at all, while WoS offers partial results. On the other hand, Pubmed allows only the export of a feature named 'Create Date' which refers to the date the record was added to the database and not the actual date of publication (Nahin 2008). Given the fact that Pubmed had the best coverage in terms of GC related publications the authors opted to retrieve all the aforementioned variables by making use of the Pubmed database.

To download the variables an algorithm using all 656 documents'Pubmed ID as input was implemented within the Python environment (3.8.5). An API key was initialized to gain access to the NCBI interactive documentation. ${ }^{2}$ Below the steps followed with respect to the variable retrieval are presented.

\section{Retrieval of document title, author names and publication dates}

Using the Pubmed ID as an input, the document title, author names and date of publication for each document was parsed (forming XPath queries). All author names and document titles were downloaded. For each retrieved date, the relevant month was extracted and saved for analysis purposes. However, in 13 out of 656 cases the actual date (month) of the publication was not provided. For such cases, further research was carried out. Specifically, given each document title, internet sources (journal homepages, related search engines) and social media (LinkedIn and Twitter) were manually examined. All 13 cases of missing months were identified.

\section{Retrieval of author affiliation and country}

To retrieve the country in which each paper was published (i.e. affiliation country) the author's affiliation needed to be extracted. This is based on the assumption that geographic location of the affiliated institution named by the author as her/his postal address stands as the country to which the specific article should be 'measured' for-this is an important assumption that cuts through the entirety of this paper. Text processing techniques such as capitalisation, stemming and lemmatization were applied in order to extract the corresponding author affiliation. Furthermore, this affiliation was parsed (forming XPath queries) to obtain the corresponding affiliation country.

By creating certain queries (utilising built-in XML parser), each document was parsed and all the aforementioned bibliometric variables were retrieved.

Within the Python (3.8.5) environment, a dictionary for each document Pubmed ID was created. This dictionary uses the document Pubmed IDs as "keys" and the Author names, Author affiliation, Month of Publication of each document as "values". Below, an example describing the structure of the finalised researcher profile is presented.

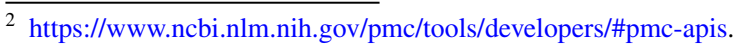


Dictionary structure \{'Pubmed ID': (Document title, Author names, Author affiliation, Country of Affiliation, Month of Publication) .

The data collection as well as variable retrieval were last updated on 23/10/2020. For the complete dataset refer to Online Appendix.

\section{Analysis}

The bibliometric data were analysed in order to perform a descriptive analysis of the scientific outputs, the national institutional sectors' contribution to the scientific output as well as the evolution of the international scientific collaboration. In view of this and in order to imprint the international scientific collaboration network, graph analysis is additionally conducted.

\section{COVID-19 related Greek publications: output and collaboration status}

With regards to descriptive analysis, as a means to measure the scientific output, the total number of publications on a monthly basis were calculated. By observing the relevant data, 5 publications had been published in a date prior to the year 2020. Since the COVID19 outbreak was originally identified in early 2020 (WHO, 2020), the authors decided to examine the context (abstracts) of these publications. The subject of analysis concerned coronaviruses-yet other types than COVID-19 types since coronaviruses have long been recognized as human pathogens (Deming \& Chen, 2020). It was decided, thus, to exclude those publications from the dataset, leading to a total yield of $651(=656-5)$ COVID-19 related publications that have been (co)authored by researchers affiliated with Greek institutions. For each publication month-that is, the month that each specific scientific publication was published (see "Variables" section) — a plot depicting the scientific activity relevant to COVID-19 publications was created. The timeframe of the analysis concerns the interval between February - when the first COVID-19 cases in Greece were identified(Worldometers, 2020a) and late October (23/10/2020).

\section{National institutional sectors: participation and contribution}

Moreover, for the purposes of understanding the national institutional sectors' contribution to the scientific output, the affiliated institutions were distributed according to the accepted institutional sectors HES, GOV, BES, $\mathrm{PNP}^{3}$ and its subclasses following OECD's Frascati taxonomy (OECD, 2015).

The above classification enabled the analysis and allowed the exploration of each institutional sector in the following twofold way: (a) in terms of institutional sector' participation to the COVID-19 related Greek scientific output, and (b) in terms of each sector's contribution relevant to the COVID-19 related Greek scientific output. Below, both ways are elucidated.

\footnotetext{
${ }^{3}$ Higher Education Sector (HES), Government Sector (GOV), Business Sector (BES), Private Non Profit Sector (PNP).
} 
1. The participation of each institutional sector is determined by calculating the frequency of the appearance of such a sector across all identified institutional affiliations (see Table 6 in "Findings" section).

2. The contribution of each institutional sector is determined by calculating the (normalised) number of publications that the specific sector is affiliated with across all identified scientific publications (see Table 6 in "Findings" section).

\section{International scientific collaboration: imprint, evolution and network}

While science has been a global collaborative endeavour (Wagner \& Graber, 2018), COVID-19 only increased this trend (Zhang et al., 2020). As a result, and in the context of this paper, it is attempted to imprint the international scientific collaboration that has been identified within the publications that have been (co)-authored by scholars affiliated with Greek institutions as well as present its evolution and network.

\section{Imprint}

To imprint the international scientific collaboration, the affiliation countries of each publication were examined. Specifically, for the purposes of the analysis, the 'country' variable had been downloaded for each publication that had been (co)-authored by a scholar affiliated with a Greek institution. Herein, each country's participation was considered unique for each publication regardless of the number of times the specific country appeared in a given publication.

For example:

Four (4) authors have (co)-authored one (1) scientific publication "X". X has been authored by researchers affiliated with the following four (4) institutional affiliations: "National and Kapodistrian University of Athens, Athens, Greece", "Department of Animal Medicine, Production and Health (MAPS), University of Padua, Italy", "School of Pharmacy, University of Camerino, Camerino, Italy" and "Department of Movement Science, Chatham University, Pittsburgh, PA, USA." The above sentence, in the context of this paper, is abbreviated as follows: The scientific publication " $\mathrm{X}$ " is affiliated with three (3) countries: Greece, Italy and the USA.

Within the context of this analysis, 101 such countries were identified.

\section{Evolution}

In order to schematically point out the evolution of the international scientific collaboration a map containing the co-affiliation countries (see subsection Retrieval of author affiliation and country) in a monthly basis manner is presented. The map was constructed taking into consideration the monthly contribution (in terms of number of publications) of each country.

Specifically, the purpose was to show the evolution of the contributors in this scientific matter and showing this evolution on a world map. After creating this chart, it is hard not to appreciate its side effects, such as easily and simply comparing the quantity of contributions of each country. For this chart, Mathematica was used-the type of chart was GeoRegionValuePlot. On a global map, this function colours the selected countries using a colouring scheme. This gets more intense (less bright) proportionately to the number of scientific publications authored from each country. 
Table 4 Paper terminology (in parenthesis) in tandem with the VOSviewer software terminology (Van Eck \& Waltman, 2018)

\begin{tabular}{ll}
\hline Term & Description \\
\hline $\begin{array}{l}\text { Items (nodes) } \\
\text { Link (edge) }\end{array}$ & $\begin{array}{l}\text { Objects of interest (countries) } \\
\text { Connection or relation between two items (co-authorship among } \\
\text { countries) } \\
\text { Attribute of each link expressed by a positive numerical value } \\
\text { Link strength }\end{array}$ \\
$\begin{array}{l}\text { (association strength) } \\
\text { Setwork }\end{array}$ & $\begin{array}{l}\text { Sets of items connected by their links } \\
\text { cluster }\end{array}$ \\
Cluster & The number of links of an item with other items \\
Weight attribute: number of links & The cumulative strength of the links of an item with other items \\
Weight attribute: total link strength
\end{tabular}

\section{Network}

Herein, the methodological framework as discussed in Sachini et al. (2020a) in the "Graph analysis" section is followed.

Specifically, for constructing the scientific connections between the target country, Greece, as well as different groups of countries, an undirected graph was constructed. Each node represents the affiliation country and each edge represents at least one coaffiliated publication from the current country to another (Table 4).

Both theoretical and empirical results indicate that co-occurrence data can best be normalized using a probabilistic measure. This provides strong support for the use of the association strength in scientometric research (Van Eck \& Waltman, 2009). Hence, the associated strength was used for normalising the co-occurrence frequencies between every pair of nodes (countries), thus meaning, the frequency of any linked pair of nodes.

For distributing the connections between the nodes (countries) a unified approach of VOS node mapping and clustering technique was selected (Waltman et al., 2010). The higher the association strength of two nodes, the stronger the attractive force between the nodes hence the closer they appear. In our case, the closer two countries are located to each other, the stronger their scientific collaboration.

In a similar manner as described in Sachini et al. (2020a), the unified methods of mapping and clustering allow us to visualise in a largely comprehensive way the international scientific collaboration of researchers. Specifically, in addition to the identification of countries with strong scientific collaborations (as a result of the application of the mapping technique), one can additionally distinguish various collaboration patterns (as a result of the application of the clustering technique). That is, groups as well as neighborhoods of countries that constitute focal points on the COVID-19 related Greek scientific publications.

The graph was constructed within the Python (3.9.0) environment, saved in an output file (.gml) and used as input for the software visualisation tool VOSviewer (Van Eck \& Waltman, 2018). 


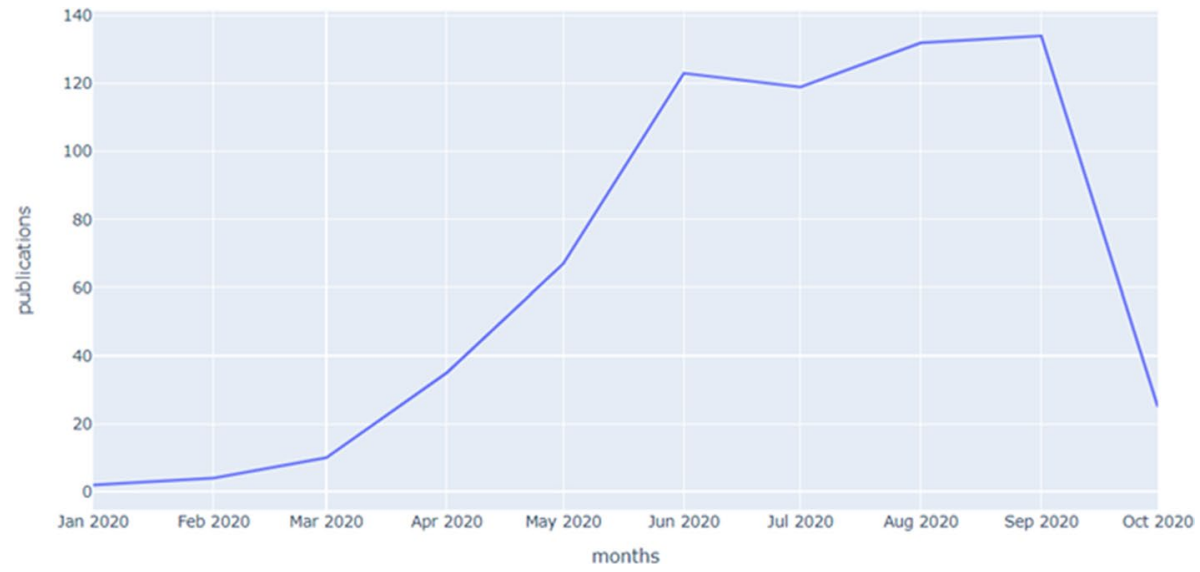

Fig. 1 Distribution of the COVID-19 Greek related publications status over time (in months)

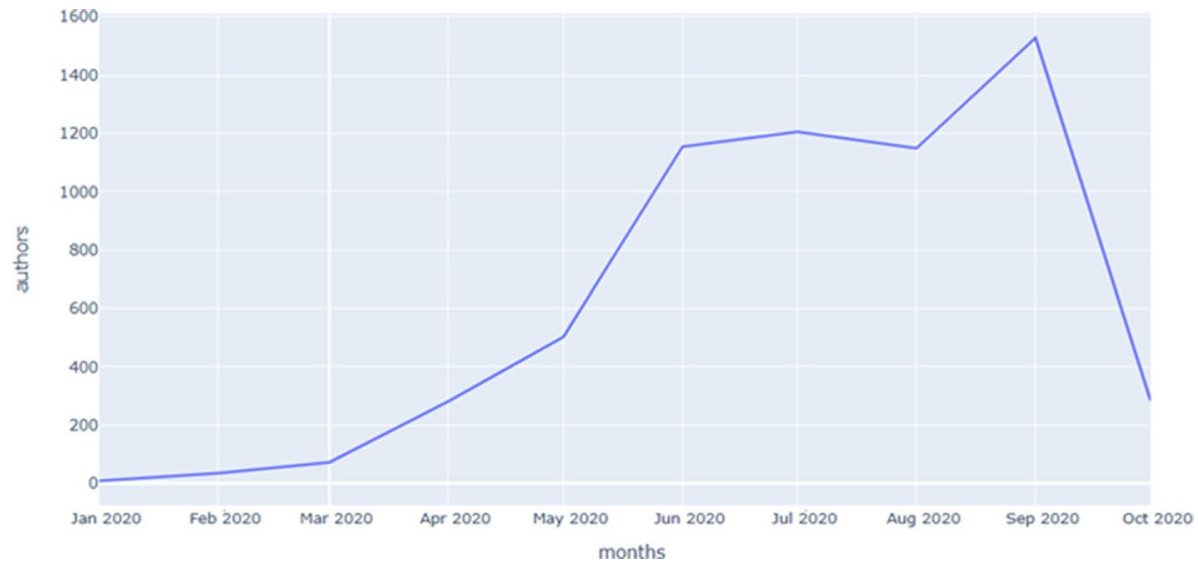

Fig. 2 Author collaboration status of COVID-19 related Greek publications over time (in months)

\section{Findings}

\section{COVID-19 related Greek publications: output and collaboration status}

As indicated above, the total number of COVID-19 related Greek publications identified amounted to 651 . Figure 1 plots the monthly trends of such publications. According to the documentation provided, the first COVID-19 related scientific article (co)authored by Greek researchers was published in January 2020. Since then, the number of publications is gradually increasing each month. With regard to the period June-July a slight decrease in the number of publications is observed (from 123 to 120). This difference can safely be neglected considering the overall publication trend. In September, scientific publications reached their global maximum (amounting to 134 documents) until decreasing for the next month (25 documents). Such a decrease can be attributed to the 
Table 5 COVID-19 related Greek publications' affiliation status

Table 6 Distribution of institutional sectors in terms of (a) sector participation and (b) sector contribution with respect to the COVID-19 Greek related scientific publications

\begin{tabular}{lll}
\hline & Frequency & Percentage \\
\hline $\begin{array}{l}\text { Publications affiliated solely } \\
\text { with Greek institutions }\end{array}$ & 285 & 43.8 \\
$\begin{array}{l}\text { Publications affiliated with } \\
\text { Greek and foreign institutions }\end{array}$ & 366 & 56.2 \\
Total Publications & 651 & 100 \\
\hline
\end{tabular}

\begin{tabular}{lllllc}
\hline & \multicolumn{2}{l}{$\begin{array}{l}\text { (a)The participation of each } \\
\text { institutional sector }\end{array}$} & & \multicolumn{2}{l}{$\begin{array}{l}\text { (b) The contribution of } \\
\text { each institutional sector }\end{array}$} \\
\cline { 2 - 3 } \cline { 5 - 6 } \cline { 5 - 6 } & Frequency & Percentage & & Frequency & Percentage \\
\hline HES & 44 & 31.4 & & 440 & 67.6 \\
GOV & 65 & 46.4 & & 176 & 27.1 \\
BES & 16 & 11.4 & & 18 & 2.7 \\
PNP & 15 & 10.7 & & 17 & 2.5 \\
Total & 140 & 100.0 & & 651 & 100.0 \\
\hline
\end{tabular}

fact that this research is conducted in late October-document recording in scientific databases regarding this period is still ongoing.

By way of comparing the two figures (Figs. 1,2), it is made evident that the increase in the number of publications is strongly correlated with the increase in the number of coauthors. This points to the mobilizing effect COVID-19 has had on researchers as well as the latter's intent on contributing (through the form of a co-authorship) to the global battle against this coronavirus.

\section{National institutional sectors: Participation and contribution}

Table 5 presents the COVID-19 related Greek publications' affiliation status. The results suggest that in terms of frequency, there are no great differences among the status of affiliated publications. Although this research scope is to focus on the scientific output related to COVID-19 on a national level, we observe that more than $50 \%$ of such output is authored through an international collaboration. This corroborates the fact that COVID-19 as a catastrophic and urgent event accelerates trends in international collaboration and requires a transnational response (Fry et al., 2020).

In Table 6 the distribution of institutional sectors in terms of (a) sector contribution and (b) sector participation intertwined with the COVID-19 related Greek scientific publications is presented (for the difference between contribution and participation, refer to section "National institutional sectors: participation and contribution").

The greatest portion of the scientific publications (67.6\%) is authored by researchers affiliated with the HES sector. That is, the HES sector significantly over-contributes when compared to the other sectors (GOV, BES, PNP) in respect to the total number of COVID-19 scientific publications. As shown in Fig. 3, Universities contribute the most scientific publications of the HES sector (51.9\%) while University Hospitals contribute 

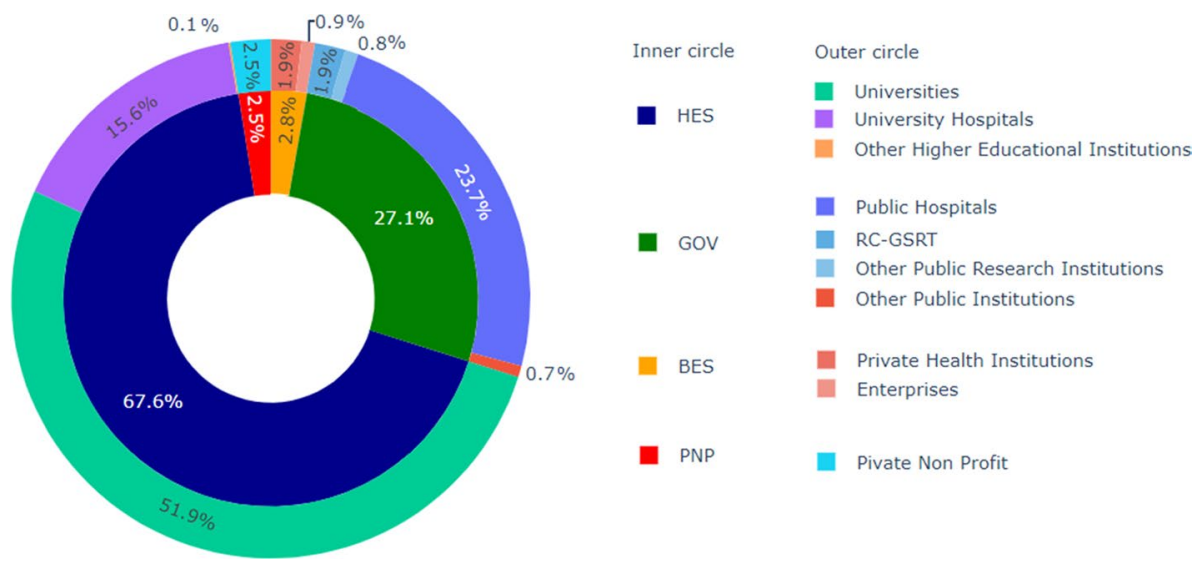

Fig. 3 Research output contribution of Greek institutional affiliations classified by sector and subsector

$15.6 \%$ of them. The limited number of medical oriented institutions is a factor that justifies the lower participation rate of the HES sector with respect to the GOV.

Despite this contribution, in terms of participation this is not the case for HES. Rather, its performance amounts to $31.4 \%$. In this case, the GOV sector has the greatest participation share with respect to the rest of the institutional sectors (46.6\%). That is, although the HES institutional sector authored the majority of scientific publications, it yields to less number of participating institutions. Conversely, in the GOV sector, more participating institutions have been mobilized to participate into related research endeavours. The above, in our view, can have twofold interpretation.

With the aim of exploring the pandemic effects, the GOV sector mobilized its full array of participating institutions, such as Public Hospitals, Research Centres supervised by the General Secretariat for Research and Technology (RC-GSRT), Other Public Research Institutions, Other Public Institutions, etc. into contributing to the global academic response against COVID-19. These subclasses of the GOV category have significant research output according to the nationwide bibliometric analysis carried out by the EKT (Sachini et al., 2020b). The significant participation of the public hospitals can be accredited to the medical oriented context relevant to COVID-19 research output. Considering the contribution level in terms of (co)authoring COVID-19 related scientific publications, in reference to the GOV sector (27.1\%, Fig. 3), public hospitals stand out (23.7\%, Fig. 3).

However, the HES sector, although with lower participation rate performed better in terms of number of research output. This can be attributed to the fact that the HES sector is inherently more involved in the research and everything pragmatic intertwined with it (scientific publications, conferences etc. compared to every other institutional sector.

Concerning BES, its participation (11.4\%) despite being low in relation to both GOV and HES, is significantly higher compared to its overall contribution $(2.8 \%)$ pointing to a select few pharmaceutical and biological firms that have been competent enough to participate in a number of such science outputs with a view on capitalizing this know how for putting out COVID-19-related products and services. 


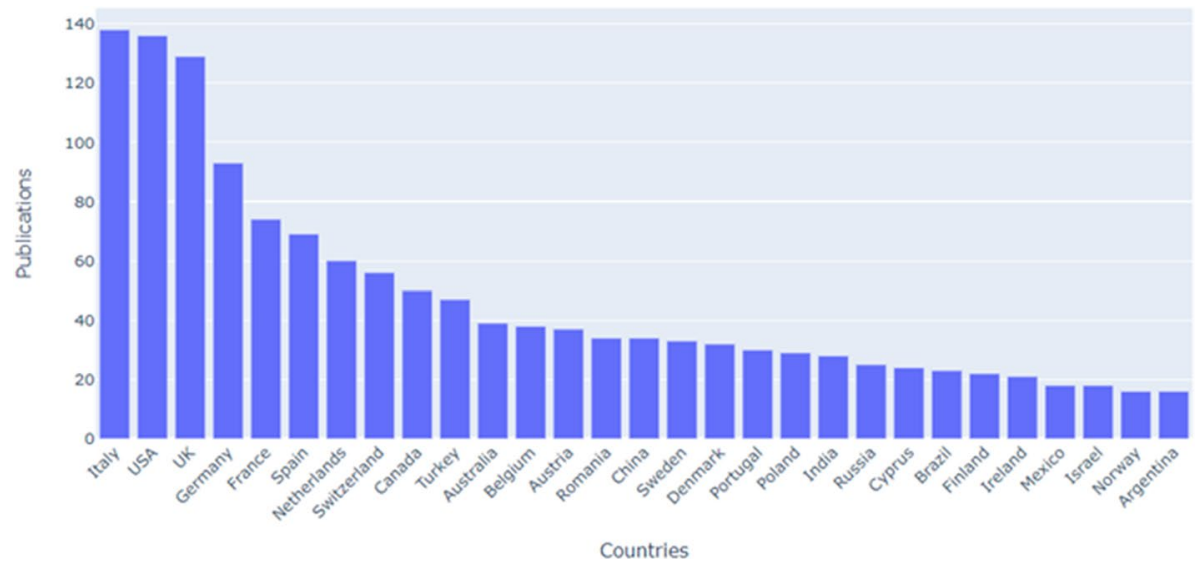

Fig. 4 The imprint of the international scientific collaboration. Distribution of the country's participation (in terms of co-occurrence frequency with the target) to the scientific publications (top 30)

\section{International scientific collaboration: imprint, evolution and network}

\section{Imprint}

Figure 4 presents the top 30 countries in terms of co-occurrence frequency in the number of scientific publications. Among the countries that participated the most in the publications that have been (co)authored by researchers affiliated with Greek institutions are: Italy, USA, the UK, Germany, France and Spain. That is, researchers affiliated with institutions in those countries.

Considering the intersection with the top 10 countries of the country list as displayed in Fig. 4 and those top 10 countries addressed by SCImago Journal selecting the subject area of 'Medicine'" (United States, United Kingdom, Germany, Canada, Japan, France, Italy, Netherlands, Australia, China) it appears that the majority of publications is affiliated with countries having 'traditionally' significant scientific impact on the field of Medicine. Specifically, it can be concluded that each of the following countries; Italy, USA and the UK (co)authored almost 20\% (135, 134 and 125 publications respectively) of the COVID-19 related Greek scientific publications.

\section{Evolution}

Figure 5 presents evidence of the monthly evolution of the international scientific collaboration network of co-authors in those scientific publications that have been (co)authored by researchers affiliated with Greek institutions. This monthly analysis allows the reader to explore the pattern of collaboration at the country level; the colour in each country corresponds to the number of publications for the specific month that corresponds to each chart. The colour intensity (yellow, orange and red) is analogous with the number of scientific

${ }^{4}$ https://www.scimagojr.com/countryrank.php?order=ci\&ord=desc\&area=2700, accessed 23/10/2020. 

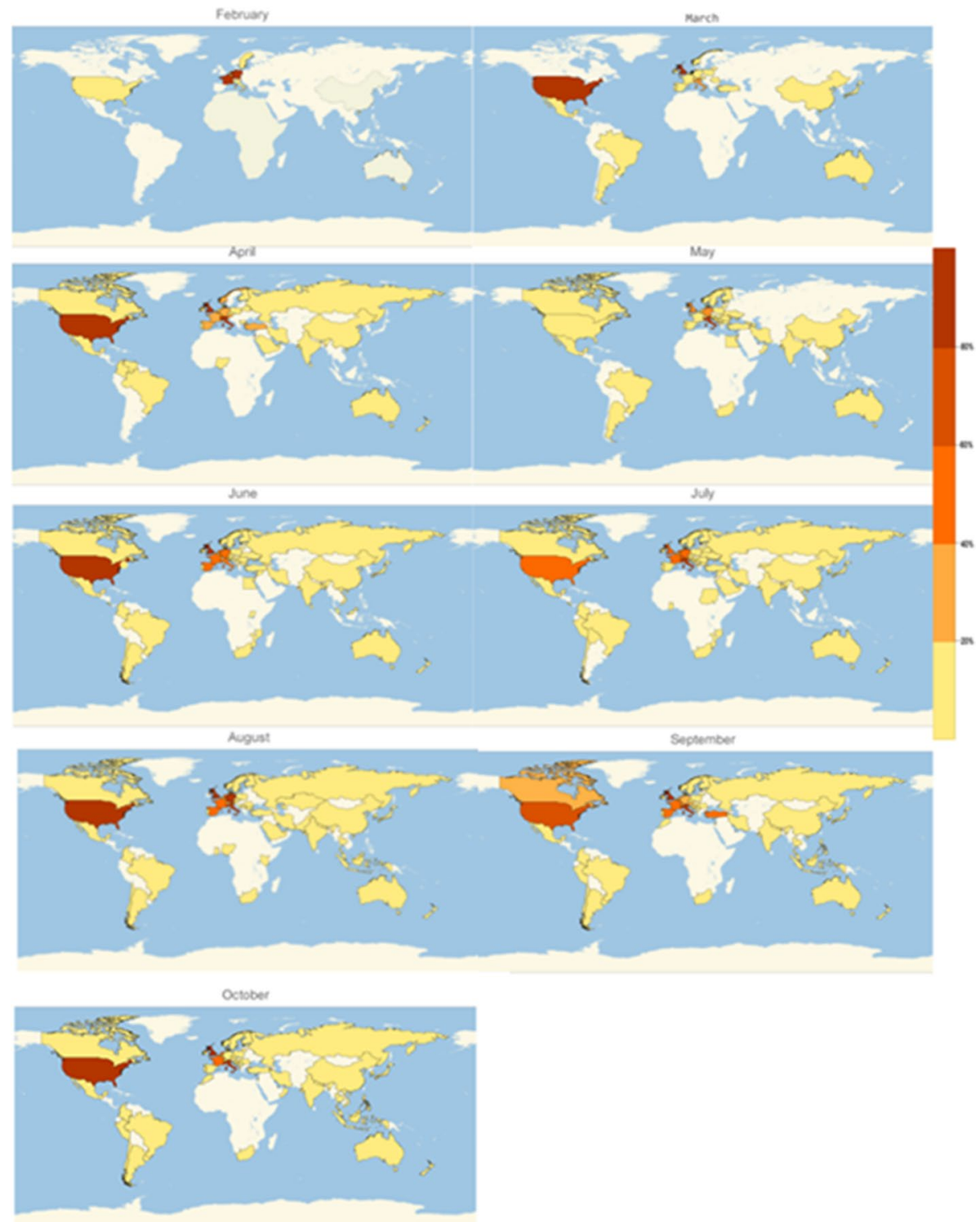

Fig. 5 The international scientific collaboration evolution

publications authored by each country; the more intense colour the greater number of scientific publications (see scale, Fig. 5).

Since the early months of 2020 (February, March) when the international community first received notice of the pandemic (WHO, 2020), scientific publications had been authored mostly by researchers affiliated with intra-EU institutions. US contribution lagged behind (Fig. 5). As realisation of the global consequences of the pandemic is reached, non-EU countries begin to co-author. This is mostly the case with the USA, 


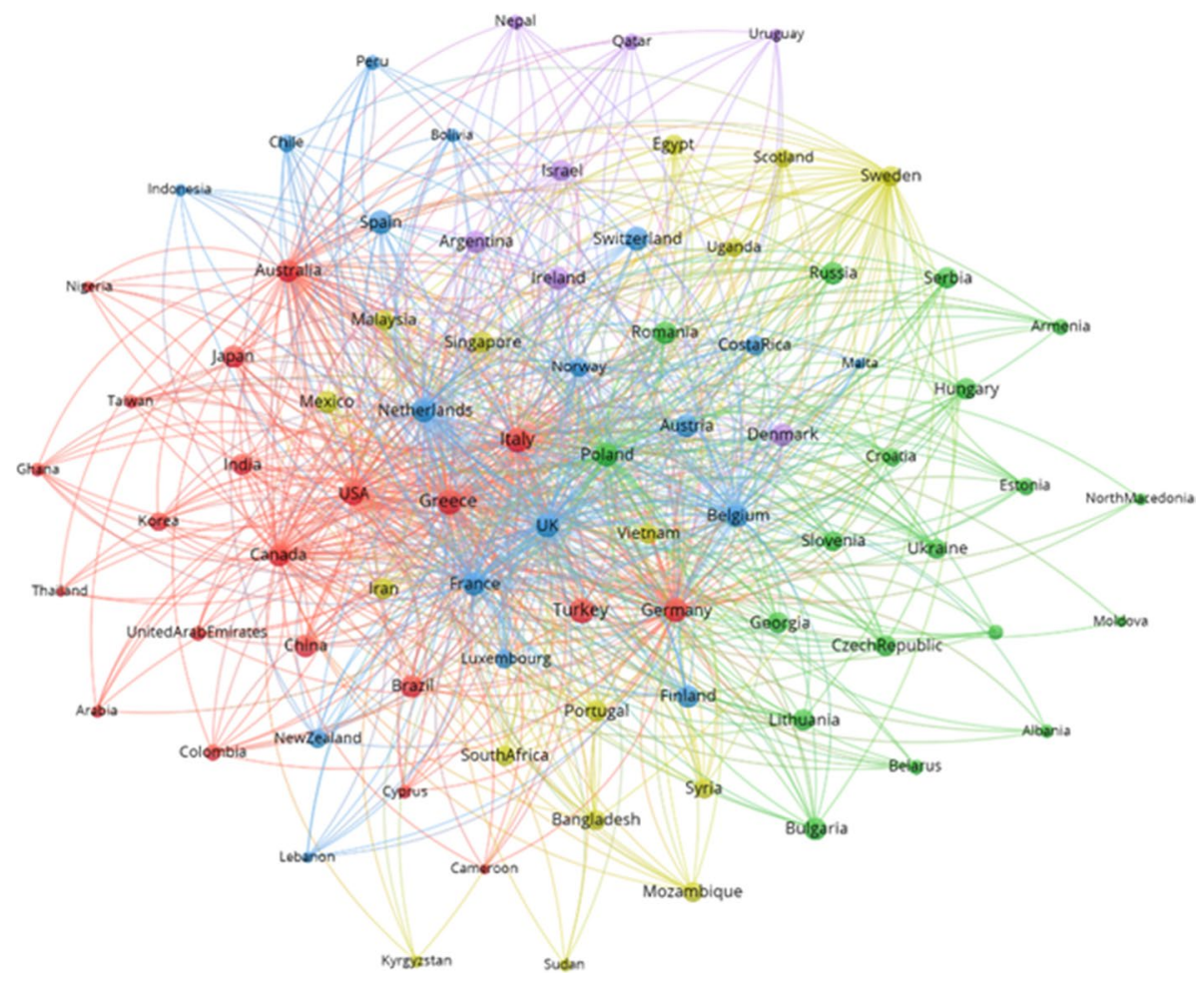

Fig. 6 The international scientific collaboration network

Canada and Central Europe (including the UK). These countries become steady coauthoring contributors from then on. Few other countries such as Asian (China, Japan, Indonesia, Thailand) and African ones (South Africa, Cameroon, Ghana, Nigeria), South America (Brazil, Argentina, Uruguay, Colombia) \& Australia (New Zealand) sporadically co-author publications.

Validating findings by Lee \& Haupt (2020) and Zhang et al. (2020), the exploited dataset in this paper indicates that as time goes on, more countries are added to the international collaboration map (peaking in September) while the colouring becomes more intense. This is indicative of the steady increase in the number of collaboration countries as well as the number of scientific contributions (in terms of number of publications) attributed to each country.

It is to be noted that Greece remains colourless in all eight (8) maps on purpose since the authors opted to imprint the evolution of the international collaboration. The target country, Greece, was taken as a given.

\subsubsection{Network}

The collaboration network analysis of countries provided 101 results. Applying a threshold of a minimum of 20 documents published per country (around 3\% of the total number of publications), 81 countries were selected. The analysis generated five different clusters (shown in yellow, red, green, purple and blue). Every cluster depicts the scientific connections between the collaboration countries (Fig. 6). Considering the inherent sparsity 
Table 7 Distribution of the top 5 countries according to their total link strength

\begin{tabular}{ll}
\hline Total link strength & Countries \\
\hline Italy & 79 \\
Turkey & 77 \\
UK & 75 \\
Germany & 74 \\
Spain & 72 \\
\hline
\end{tabular}

of the co-authorship network, the attraction and repulsion parameters were set to 2 and - 2 respectively (Van Eck \& Waltman, 2015).

The first five (5) countries based on total link strength are shown in Table 7. These constitute the top five countries with the most connections among all the countries in the international scientific collaboration network. Considering Europe, such countries have the greatest number of total COVID-19 cases among the rest of the European countries (Worldometers, 2020b). This indicates a positive correlation between the number of scientific publications and the COVID-19 total cases at a European level.

By visualising Fig. 6, observing the countries' positioning in the network, and being guided as well by the node size, it is evident that countries such as Italy, USA, the UK, France and the Netherlands are examples of countries which collaborated the most with Greece with respect to the rest of countries. In reference to Fig. 4, these are countries with the greatest participation in the COVID-19 related publications, in terms of co-occurrence frequency with the target. Such countries have a high association strength with Greece indicating a high degree of similarity (Van Eck \& Waltman, 2014). The word "similarity" in this study should be interpreted in terms of the amount of co-authoring scientific publications related to the COVID-19 pandemic.

On the other hand, countries such as Nepal (purple cluster), Indonesia (blue cluster), Thailand (red cluster), Kyrgyzstan (yellow cluster) and Moldova (green cluster), are countries which collaborated the least with Greece with respect to the rest of the countries. ${ }^{5}$

\section{Discussion}

This study attempted to imprint the scientific outputs related to the COVID-19 pandemic that have been (co)authored by the science base at the country level. In addition, it sought to highlight the respective international level of collaboration. In relation to the research findings, the number of publications as well as the number of scientific collaborations indicates a steady increase over time. At a cross-country level, affiliated institutional sectors such as HES and GOV contributed the most in terms of scientific output.

On an international scale, the evolution of scientific collaboration reveals a significant, increasing author participation over time. Overall, 101 different countries have been involved in (co)authoring these publications. This corroborates the fact that the pandemic and its effects increased the international collaboration trend (Fry et al., 2020; Zhang et al., 2020).

\footnotetext{
5 The term "with respect to the rest of the countries", in a quantitative manner, is justified and interpreted by the selection of the association strength as a probabilistic normalising measure (see section "Network").
} 
The scientific collaboration is alternatively distributed in terms of a connected coauthorship network. Such a network depicts a chain of affiliations wherein nations are linked together. Such chains are represented as clusters of countries, in which the scientific connections between different countries can be visualised. In terms of countries with the most scientific connections among all the countries in the international scientific collaboration Italy, Turkey, the UK, Germany and Spain stand out.

With regard to the COVID-19 related Greek scientific publications, it can be reasoned that a significant amount of them $(20 \%)$ is affiliated with countries having "traditionally" major scientific impact on the field of Medicine.

\section{Further research}

Exploring the networking evolution of other countries based on the co-authoring pattern of their science base is a potential avenue for further research-especially in those instances where a highly performing human capital and/or a strong in terms of science output country is the case.

The hypothesis that the co-authoring patterns have been initiated ex nihilo due to the urgency imposed by the need to come up with a solution against COVID-19 or were built upon existing research collaboration is something that can be tested. This a potential further avenue that can be tested in the case of Greek researchers.

Similarly, exploring the co-authoring patterns of researchers dependent on sex and professorial and researcher level is of interest, in addition to examining the correspondence between standard bibliometric indicators to alternative ones, such as bookmarks on reference managers like Mendeley, and mentions on social networks such as Twitter.

Supplementary Information The online version contains supplementary material available at https://doi. org/10.1007/s11192-021-03952-9.

\section{References}

Benjamens, S., de Meijer, V. E., Pol, R. A., \& Haring, M. P. D. (2020). Are all voices heard in the COVID19 debate? Scientometrics. https://doi.org/10.1007/s11192-020-03730-Z

Blundell, R., Costa Dias, M., Joyce, R., \& Xu, X. (2020). COVID-19 and Inequalities. Fiscal Studies, 41(2), 291-319.

Bonaccorsi, G., Pierri, F., Cinelli, M., Flori, A., Galeazzi, A., Porcelli, F., Schmidt, A. L., Valensise, C. M., Scala, A., Quattrociocchi, W., \& Pammolli, F. (2020). Economic and social consequences of human mobility restrictions under COVID-19. Proceedings of the National Academy of Sciences, 117(27), $15530-15535$.

Collins, G. S., van Smeden, M., \& Riley, R. D. (2020). COVID-19 prediction models should adhere to methodological and reporting standards. European Respiratory Journal, 56(3), 2002643.

Deming, M. E., \& Chen, W. H. (2020). COVID-19 and lessons to be learned from prior coronavirus outbreaks. Annals of the American Thoracic Society, 17, 790-794.

Dingel, J. I., \& Neiman, B. (2020). How many jobs can be done at home? (No. w26948). National Bureau of Economic Research. Economic Bulletin Economic and monetary developments. (2020). European Central Bank, 2020.

EU Commission. (2020). Coronavirus: Commission launches one-stop shop for coronavirus research and innovation funding. Retrieved October 15, 2020 from https://ec.europa.eu/research/horizon2020/index. $\mathrm{cfm} ? \mathrm{pg}=$ country-profiles-detail\&ctry=Greece.

Fana, M., Pérez, S. T., \& Fernández-Macías, E. (2020). Employment impact of Covid-19 crisis: From short term effects to long terms prospects. Journal of Industrial and Business Economics, 47(3), 391-410. 
Fraizer T., Auvil S. (2020) Patent litigation filings on the increase with the COVID-19 pandemic (2020). https://www.iptechblog.com/2020/05/patent-litigation-filings-on-the-increase-with-the-covid-19pandemic/.

Fry, C. V., Cai, X., Zhang, Y., \& Wagner, C. S. (2020). Consolidation in a crisis: patterns of international collaboration in early COVID-19 research. PLoS ONE, 15(7), e0236307.

Homolak, J., Kodvanj, I., \& Virag, D. (2020). Preliminary analysis of COVID-19 academic information patterns: a call for open science in the times of closed borders. Scientometrics, 124(3), 2687-2701.

Horbach, S. P. (2020). Pandemic publishing: Medical journals strongly speed up their publication process for Covid-19. Quantitative Science Studies, 1(3), 1056-1067.

Horizon (2020). Horizon 2020 country profile for Greece.Country participation and featured projects for Greece. European Commission. Retrieved October 12,2020 from https://ec.europa.eu/info/news/coron avirus-commission-launches-one-stop-shop-coronavirus-research-and-innovation-funding-2020-may14_en.

Hossain, M. M. (2020). Current status of global research on novel coronavirus disease (Covid-19): A bibliometric analysis and knowledge mapping. SSRN Electronic Journal. https://doi.org/10.1787/97892 64239012-en

Kambhampati, S. B., Vaishya, R., \& Vaish, A. (2020). Unprecedented surge in publications related to COVID-19 in the first three months of pandemic: A bibliometric analytic report. Journal of Clinical Orthopaedics and Trauma, 11(Suppl 3), S304.

Kokol, P., \& Vošner, H. B. (2018). Discrepancies among Scopus, Web of Science, and PubMed coverage of funding information in medical journal articles. Journal of the Medical Library Association: JMLA, $106(1), 81$.

Kousha, K., \& Thelwall, M. (2020). COVID-19 publications: Database coverage, citations, readers, tweets, news, Facebook walls, Reddit posts. Quantitative Science Studies, 1-28 (Just Accepted).

Lee, J. J., \& Haupt, J. P. (2020). Scientific globalism during a global crisis: Research collaboration and open access publications on COVID-19. Higher Education, 1-18. https://doi.org/10.1007/ s10734-020-00589-0.

Marc Botenga (2020) European Parliament. Subject: COVID-19 vaccine as a public good. Retrieved October 22,2020

Mariolis, T., Rodousakis, N., \& Soklis, G. (2020). The COVID-19 multiplier effects of tourism on the Greek economy. Tourism Economics, 1354816620946547.

Mercer University. (2020). Difference between PubMed, Embase, Web of Science and Scopus. Retrieved October 22, 2020 from https://med.mercer.edu/library/database-differences.htm.

Nahin, A. (2008). Create date-New field indicates when record added to PubMed. NLM tech.

Natur. (2020). Coronavirus: everyone wins when patents are pooled. Nature, 581(7808), 240. https://doi.org/ 10.1038/d41586-020-01441-2

NSB. (2019). Publication output: US trends and international comparisons. Science and Engineering Indicators 2020.

OECD. (2015). Frascati Manual 2015: Guidelines for Collecting and Reporting Data on Research and Experimental Development, The Measurement of Scientific, Technological and Innovation Activities. OECD Publishing.

OECD. (2020a). Using artificial intelligence to help combat COVID-19. OECD Publishing Paris. Retrieved October 20, 2020 from https://read.oecd-ilibrary.org/view/?ref=130_130771-3jtyra9uoh\&title=Usingartificial-intelligence-to-help-combat-COVID-19.

OECD. (2020b). A framework to guide an education response to the COVID-19 Pandemic of 2020, Organisation of Economic Cooperation and Development, Paris. Retrieved October 20, 2020 from https:// read.oecd-ilibrary.org/view/?ref=126_126988-t631xosohs\&title=A-framework-to-guide-an-educationresponse-to-the-Covid-19-Pandemic-of2020\&utm_source=Adestra\&utm_medium=email\&utm_conte $\mathrm{nt}=$ Learn $\% 20$ more\&utm_campaign=OECD $\% 20$ Education $\% 20 \% 26 \% 20$ Skills $\% 20$ Newsletter\%3A\% 20April\%202020\&utm_term=demo.

OECD. (2020c). Why open science is critical to combatting COVID-19. Organisation of Economic Cooperation and Development. Retrieved October 20, 2022 from https://read.oecd-ilibrary.org/view/?ref= 129_129916-31pgjnl6cb\&title=Why-open-science-is-critical-to-combatting-COVID-19.

OPSI (2020). COVID-19 innovative response tracker. Retrieved October 22, 2020.

Paaso, A., Bahramirad, S., Beerten, J., Bernabeu, E., Chiu, B., Enayati, B., Hederman, B., Jones, L., Jun, Y., Koch, H., Montero, J. C., Nair, N., Novosel, D., Pierpoint, T., Rahmatian, F., Aguero, J. R., Root, C., Sharafi, D., Tejera, E., \& Vittal, V. (2020). Sharing knowledge on electrical energy industry's first response to COVID-19.

Pai, M. (2020). Covidization of research: What are the risks? Nature Medicine, 26(8), 1159-1159. 
Palayew, A., Norgaard, O., Safreed-Harmon, K., Andersen, T. H., Rasmussen, L. N., \& Lazarus, J. V. (2020). Pandemic publishing poses a new COVID-19 challenge. Nature Human Behaviour, 4(7), $666-669$.

Prudêncio, M., \& Costa, J. C. (2020). Research funding after COVID-19. Nature Microbiology, 5, 986. https://doi.org/10.1038/s41564-020-0768-Z

Sachini, E., Karampekios, N., Brutti, P., \& Sioumalas-Christodoulou, K. (2020a). Should I stay or should I go? Using bibliometrics to identify the international mobility of highly educated Greek manpower. Scientometrics, 125(1), 641-663.

Sachini, E., Malliou, N., Chrysomallidis, C., Karampekios, N. (2020b), Greek Scientific Publications 20042018: Bibliometric analysis of articles in peer reviewed journals-Web of Science (WoS). National Documentation Centre. Available at: https://metrics.ekt.gr/publications/435 [in Greek].

ScienceBusiness. (2020a). LIVE BLOG: R\&D response to COVID-19 pandemic. Retrieved October 4, 2020 from https://sciencebusiness.net/news/live-blog-rd-response-cvid-19-pandemic.

ScienceBusiness. (2020b). Canada to boost COVID-19 research by C\$1B. Retrieved October 4, 2020 from https://sciencebusiness.net/news/canada-boost-covid-19-research-c1b.

Van Eck, N. J., \& Waltman, L. (2009). How to normalize cooccurrence data? An analysis of some wellknown similarity measures. Journal of the American Society for Information Science and Technology, 60(8), 1635-1651.

Van Eck, N. J., \& Waltman, L. (2014). Visualizing bibliometric networks. In Measuring scholarly impact (pp. 285-320). Springer.

Van Eck N.J., Waltman L. (2015). Network visualization: Fine-tuning layout techniques for different types of networks. Centre for Science and Technology Studies (CWTS), Leiden University. Fifth International Workshop on Social Network Analysis (ARS'15) Capri, Italy, April 30, 2015.

Van Eck N.J., Waltman L., (2018). Manual for VOSviewer version 1.6.8, CWTS Meaningful Metrics, Universiteit Leiden.

Visser, M., van Eck, N. J., \& Waltman, L. (2020). Large-scale comparison of bibliographic data sources: Scopus, Web of Science, Dimensions, Crossref, and Microsoft Academic. arXiv:2005.10732.

Wagner, C. S., \& Graber. (2018). Collaborative era in science. Palgrave Macmillan.

Waltman, L., Eck, N. J. V., \& Noyons, E. C. M. (2010). A unified approach to mapping and clustering of bibliometric networks. Journal of Informetrics, 4(4), 629-635.

WHO. (2020). Archived: WHO timeline-covid-19. Retrieved from Archived: Who Timeline-COVID-19. https://www.who.int/news/item/27-04-2020-who-timeline---covid-19. Accessed on 10 May 2020.

Worldometers. (2020a).Coronavirus active cases in Greece. Worldometers. Retrieved May 10, 2020 from https://www.worldometers.info/coronavirus/country/greece/.

Worldometers. (2020b). Reported cases and deaths by country or territory. Worldometers. Retrieved October 21, 2020 from https://www.worldometers.info/coronavirus/.

Yuret, T. (2017). An analysis of the foreign-educated elite academics in the United States. Journal of Informetrics, 11(2), 358-370.

Zhang, L., Zhao, W., Sun, B., Huang, Y., \& Glänzel, W. (2020). How scientific research reacts to international public health emergencies: a global analysis of response patterns. Scientometrics, 124, 747-773.

Zhu, J., \& Liu, W. (2020). A tale of two databases: the use of Web of Science and Scopus in academic papers. Scientometrics, 1-15. 